\title{
Balance de poderes legislativos en Chile. ¿Presidencialismo exagerado o base de un sistema político cooperativo?
}

\author{
CRistóbal AninAT
}

\section{Resumen}

Este artículo sostiene que la clasificación de los poderes presidenciales utilizada en la creciente literatura sobre legislaturas latinoamericanas no permite discriminar entre sistemas legislativos conducentes a la cooperación política y sistemas legislativos en que el Ejecutivo puede modificar el status quo unilateralmente.

El análisis propuesto responde a la paradoja planteada por Siavelis (2002), según la cual Chile tendría un presidencialismo exagerado con Presidentes moderados. Se concluye que el Presidente de Chile tiene los poderes constitucionales suficientes para controlar la agenda legislativa, pero no goza de facultades que le permitan modificar el status quo unilateralmente. Esto último, junto a la existencia de quórum especiales para un número significativo de materias de ley y al sistema electoral binominal, configuran un proceso legislativo conducente a la cooperación política.

\begin{abstract}
This article argues that the classification of presidential powers developed in the recent literature on Latin American legislatures does not allow to discriminate between legislative systems conducive to political cooperation and those in which the executive is able to modify the status quo unilaterally.

The proposed framework of analysis responds to the paradox put forward by Siavelis (2002), in which Chile has moderate presidents notwithstanding having an exaggerated presidential system. The paper concludes that the constitutional powers of the Chilean president allow him to control the legislative agenda, but that he does not enjoy the authority to unilaterally change the status quo. Which, together with high supermajorities required for a considerable portion of the legislative space and the binomial electoral system, configure a cooperative legislative system.
\end{abstract}

Palabras Clave: Chile - instituciones políticas - proceso legislativo - balance de poderes cooperación política.

\section{Introducción}

Durante décadas, el estudio de las legislaturas latinoamericanas estuvo rezagado en relación a sus equivalentes de Europa y Estados Unidos. Debido a la inestabili- 
dad política de la región, la investigación académica en ciencia política se enfocó en estudiar los quiebres democráticos, dictaduras y transiciones a la democracia. En los noventa, sin embargo, con la consolidación de los regimenes democráticos en la región, ha surgido un renovado interés por el estudio de las legislaturas latinoamericanas.

Los primeros estudios que se realizaron sobre estas legislaturas enfatizaron la preponderancia de los Presidentes en el proceso legislativo. En los estudios de Shugart y Carey (1992), Mainwaring y Shugart (1997), las legislaturas latinoamericanas fueron caracterizadas como instituciones secundarias que sólo daban legitimidad democrática al actuar del Ejecutivo (rubber stamp legislatures). Estudios posteriores enfatizaron que si bien las legislaturas latinoamericanas no tenían un rol proactivo importante como el Congreso norteamericano, éstas cumplían un rol reactivo que podía ser obstruccionista de la acción del Presidente o contribuir a la formación de políticas pública, cumpliendo así con un rol fiscalizador de la administración del gobierno ${ }^{1}$.

La literatura señalada establece que el balance de poderes entre el Presidente y el Congreso está dado por: i) el balance de facultades constitucionales del Presidente y el Congreso, ii) el contingente del Presidente en el parlamento, y iii) la capacidad legislativa del Congreso y los parlamentarios. La interacción entre estos tres elementos determina el rol del Ejecutivo y del Congreso en el proceso legislativo, el nivel de cooperación entre estos poderes, y entre los actores políticos con representación parlamentaria ${ }^{2}$.

En este contexto, Shugart y Carey (1992) y Mainwaring y Shugart (1997) clasifican los poderes presidenciales como proactivos y reactivos. Los primeros son los que le permiten al Presidente cambiar unilateralmente el status quo; los segundos le confieren la capacidad de preservar el status quo a pesar de los esfuerzos realizados por la legislatura por modificarlo. Entre los poderes proactivos del Presidente se encuentran los poderes de gobernar por decreto, los poderes de agenda (urgencias) y los poderes presupuestarios. Los poderes reactivos incluyen la iniciativa legislativa exclusiva, los poderes de veto, y la iniciativa de llamar a plebiscito. Este marco de análisis ha sido ampliamente adoptado por la literatura de corriente principal (mainstream) ${ }^{3}$.

Sin embargo, como plantea Morgenstern (2002), aunque este marco ha contribuido enormemente al estudio de los presidencialismos latinoamericanos, asume la misma importancia relativa entre los poderes presidenciales, equiparando la influencia de los poderes de decreto y los de agenda. Más aún, en este marco de análisis se considera que la facultad de controlar la agenda es un poder que permite al Presidente cambiar el status quo unilateralmente. El control de la agenda es una facultad importante del Presidente de la República, pero la aprobación

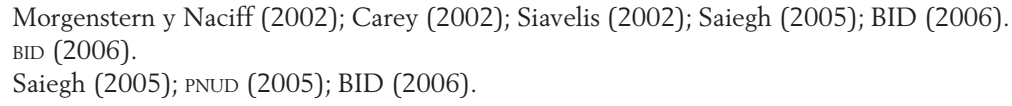


de dicha agenda requiere de una mayoría parlamentaria favorable. Distinto es el caso de la facultad del Presidente de dictar decretos sin previa autorización del Congreso o del poder de veto constructivo, el cual no necesita ser votado para su aprobación. Ambas facultades permiten a un Presidente de minoría imponer cambios al status quo que la mayoría del Congreso no aprueba.

De esta manera, este marco de análisis no ha sido suficiente para explicar por qué algunos Presidentes latinoamericanos han gobernado en prescindencia del Congreso y otros, en cambio, han mantenido una práctica política cooperativa. En este artículo se sostiene que la clasificación más relevante de los poderes del Presidente de la República en el proceso legislativo no se basa en lo que se ha llamado poderes proactivos o reactivos, sino en si sus facultades legislativas le permiten al Ejecutivo modificar el status quo unilateralmente o si sólo le otorgan la capacidad de determinar la agenda legislativa y bloquear cambios propuestos por el Congreso.

Clasificar los poderes presidenciales en función de si éstos le permiten al Ejecutivo modificar el status quo unilateralmente o si sólo le permiten manejar la agenda (u obstruir la agenda del Congreso), permite evaluar la capacidad real de un Presidente de avanzar en su agenda legislativa sin contar con mayorías en el Congreso. Esta es la variable fundamental en la relación Ejecutivo-Legislativo: es ella la que explica que algunos sistemas políticos en la región exhiban considerables niveles de cooperación entre el Presidente y el Congreso, como es el caso de Chile, mientras que otros cuenten con Presidentes que, como Carlos Menem, pueden legislar de forma unilateral.

La capacidad del Ejecutivo de prescindir del Congreso está determinada tanto por las facultades constitucionales formales y los poderes partidarios, como por la posibilidad de encontrar loopholes en el sistema institucional que rige el proceso legislativo. Como consecuencia de ello, el modelo que se propone no se basa solamente en constatar la existencia de facultades formales, sino también en identificar cómo los detalles de estas facultades interactúan para determinar un sistema de incentivos y oportunidades para la acción política del Presidente y de los parlamentarios.

El caso de Chile es particularmente relevante para nuestro argumento. En los ya clásicos análisis de Shugart y Carey (1992) y de Mainwaring y Shugart (1997), o en Siavelis (2002) y en PNUD (2005), no se logra comprender cómo un país que en las comparaciones regionales es considerado como uno de los más presidencialistas, incluso con un presidencialismo exagerado, es el país que exhibe el sistema político más cooperativo de la región (BID, 2006) con una trayectoria de Presidentes moderados desde 1990 (Siavelis, 2002).

En lo que sigue, examinaremos el marco de análisis basado en los poderes proactivos y reactivos del Presidente de la República, planteando luego un modelo alternativo. En la sección tres, analizamos el sistema institucional que rige el proceso legislativo chileno a la luz del marco de análisis propuesto en la sección anterior. En la sección cuatro concluimos. 


\section{Balance de poderes en el proceso legislativo}

La literatura comparada de corriente principal sobre el estudio de las legislaturas latinoamericanas y su poder relativo frente al de los Presidentes se basa en el planteamiento original de Shugart y Carey (1992) sobre los poderes proactivos y reactivos del Ejecutivo. Los primeros le permiten al Presidente cambiar unilateralmente el status quo; los segundos le confieren la capacidad de preservar el status quo, a pesar de los esfuerzos realizados por la legislatura por modificarlo ${ }^{4}$. Entre los poderes proactivos del Presidente se encuentran los poderes de gobernar por decreto, los poderes de agenda (urgencias) y los poderes presupuestarios. Los poderes reactivos incluyen la iniciativa legislativa exclusiva, los poderes de veto, y la iniciativa de llamar a plebiscito.

Este marco de análisis ha sido ampliamente adoptado por la literatura especializada ${ }^{5}$. Sin embargo, esta clasificación de los poderes legislativos del Presidente tiende a obscurecer el real alcance de sus atribuciones y el balance de poder resultante entre el Presidente y el Congreso. Bajo este marco de análisis, el BID (2006) plantea que los mayores poderes legislativos de los Presidentes en América latina se encuentran en Chile, Brasil, Ecuador y Colombia, y que el Presidente chileno es el que goza de mayores poderes reactivos. Así también, en el PNUD (2005), el Presidente de Chile aparece con poderes legislativos más amplios que los de Argentina. Sin embargo, como veremos más adelante, el Presidente argentino puede modificar el status quo unilateralmente, aún teniendo minoría parlamentaria. En contraste con lo anterior, el Presidente de Chile no tiene herramientas para modificar el status quo si no cuenta con mayoría en ambas cámaras del Congreso. Más aún, como resultado de la interacción de las facultades constitucionales del Presidente, las características del proceso legislativo y el sistema electoral vigente en Chile, para hacer modificaciones al status quo en las materias de ley más relevantes, el Presidente chileno necesita contar con el concurso de la oposición, lo que refuerza el poder del Congreso en el proceso legislativo.

Como se señaló anteriormente, este enfoque clasifica como poderes que permiten el cambio unilateral del status quo a los poderes de urgencia y de decreto, y considera a la vez como poderes reactivos a la iniciativa exclusiva y los poderes de veto. Sin embargo, bajo ciertos arreglos institucionales, el poder de veto constructivo permite a un Presidente de minoría imponer su versión preferida de un proyecto de ley. Por otra parte, los poderes de urgencia, no permiten bajo ninguna circunstancia la aprobación de su agenda legislativa a un Presidente de minoría.

Como lo plantea Morgenstern (2002), es necesario contar con estudios más detallados de las configuraciones constitucionales y legales que establecen el sistema legislativo de los países latinoamericanos para determinar el verdadero balance de poder entre el Presidente y el Congreso. Sólo así, podremos entender los 
efectos de estas configuraciones sobre el comportamiento de los actores políticos. Como plantea Aninat et al. (2006), la efectividad del diseño institucional está en sus detalles, los cuales determinan la posibilidad de encontrar loopholes que permitan evadir los frenos y contrapesos establecidos, que es lo que al final determina los reales incentivos de comportamiento de los actores políticos.

Los recientes trabajos de Alemán (2003); Alemán y Tsebelis (2005); Aninat y Londregan (2005 y 2006); y Londregan (2000), han contribuido en esta dirección develando aspectos más precisos del funcionamiento de los sistemas legislativos de Argentina, Brasil, Chile, Colombia y México. En estos estudios se aprecia cómo los efectos de pequeñas diferencias en los sistemas institucionales entre aquellos países, muchas veces aparentemente nominales, tienen consecuencias significativas sobre la dinámica real del poder y el balance de poder entre el Ejecutivo y el Legislativo.

En este sentido, los poderes que realmente dan al Ejecutivo la capacidad de modificar el status quo sin contar con una mayoría en el Congreso son los poderes de decreto, cuando éstos no deben ser conferidos antes de su aplicación por una mayoría parlamentaria; la iniciativa de llamar a plebiscito para aprobar legislación sin la necesidad de llevarla a votación en el Congreso; y los poderes de veto constructivo, cuando permiten que el Presidente pueda legislar con un apoyo minoritario en el Congreso. El resto de las atribuciones mencionadas dan poder al Presidente para determinar la agenda, o para modificar el status quo siempre que cuente con mayoría parlamentaria. En este contexto, la clasificación de facultades legislativas constitucionales que mejor explica el balance de poderes legislativos entre el Presidente y el Congreso es la siguiente: i) facultades que permiten al Presidente controlar la agenda legislativa (su contenido y velocidad de tramitación), ii) facultades que permiten al Presidente modificar el status quo unilateralmente; y iii) facultades que le permiten bloquear cambios al status quo impulsados por el Congreso Nacional.

En la primera categoría se encuentran las facultades de urgencias y los derechos especiales del Presidente durante el proceso de enmienda, además de la iniciativa exclusiva en ciertas materias de ley, y del control sobre la elaboración y proceso de aprobación legislativa del presupuesto de la nación. En la segunda categoría, están la facultad de dictar decretos con fuerza de ley sin autorización previa del Congreso, la iniciativa de convocar a plebiscitos, y ciertos tipos de poder de veto constructivo. El veto absoluto (total) permite al Presidente bloquear cambios al status quo aprobados por el Congreso, y la iniciativa exclusiva del Presidente en ciertas materias de ley impide que el Congreso modifique el status quo en dichas materias.

Si analizamos el proceso legislativo chileno bajo este marco conceptual, la apreciación del balance de poderes legislativos entre el Congreso y el Presidente de la República es diferente a lo que sostiene la literatura comparada de corriente principal. Este marco de análisis explica la paradoja planteada por Siavelis (2002), en que éste constata el éxito legislativo en Chile durante los años noventa a pesar de tener una institucionalidad que se considera inadecuada. Asimismo, 
este análisis de la institucionalidad legislativa chilena entrega nueva información que refuerza los resultados obtenidos por BID (2006) y Aninat et al. (2007) en relación a las bases institucionales de los altos grados de cooperación política que ha experimentado Chile desde el retorno a la democracia.

\section{Bases institucionales de un sistema legislativo cooperativo}

En esta sección se analizan, en perspectiva comparada, las bases institucionales del sistema legislativo chileno. Se analizan los poderes legislativos del Presidente de la República, las variables que determinan el nivel de influencia del Congreso en el proceso legislativo, y las variables que determinan el balance de poderes entre el Presidente y el Congreso.

\section{Poderes legislativos del Presidente de la República}

\section{Poderes de agenda: Iniciativa exclusiva, control presupuestario, y urgencias legislativas}

La Constitución Política de Chile establece que sólo el Ejecutivo puede iniciar legislación en materias relacionadas con las divisiones políticas y administrativas del país, así como su administración financiera y presupuestaria ${ }^{6}$; también sobre la contratación de deuda pública y de impuestos de cualquier naturaleza, y sobre materias laborales y de seguridad social ${ }^{7}$. Según la Constitución, el Congreso sólo podrá aceptar, disminuir o rechazar las distintas iniciativas del Ejecutivo en estas materias ${ }^{8}$. Estas disposiciones significan una restricción importante a la capacidad del Congreso para iniciar legislación, y dan iniciativa exclusiva al Presidente de la República sobre la mayor parte de la política económica de la nación, materias laborales y de seguridad social.

En los diferentes sistemas legislativos latinoamericanos se observan dos tipos de poderes de urgencia: i) la urgencia como una herramienta para determinar la agenda, y ii) una versión más fuerte en que la urgencia además determina el período de tiempo en el cual la legislatura debe pronunciarse sobre los proyectos de ley calificados de urgentes. Un ejemplo del primer caso es Brasil en que si la legislatura no se hace cargo del proyecto calificado con urgencia dentro de 45 días, éste tiene precedencia sobre cualquier otro asunto legislativo. Otro ejemplo es Colombia, donde los proyectos calificados con urgencia toman precedencia inmediata sobre toda otra legislación? ${ }^{9}$.

6 También son de iniciativa exclusiva del Presidente las normas relativas al tamaño y la disposición de las Fuerzas Armadas.

7 Este poder se ve fortalecido por el artículo 24 de la Ley Orgánica Constitucional del Congreso, que establece que no son admisibles las indicaciones de los parlamentarios que traten de materias de iniciativa exclusiva del Presidente.

CPR, artículo 65.

9 Morgenstern (2002). 
La Constitución de Chile otorga al Presidente de la República la facultad de introducir urgencias legislativas en todo momento de la tramitación legislativa de un proyecto de ley. Las urgencias pueden ser de tres tipos -urgencia simple, suma urgencia o discusión inmediata-, y ellas obligan a la Cámara en que se encuentra el proyecto a votar la iniciativa en 30 días, 10 días y 3 días respectivamente. Si el proyecto se encuentra en Comisión mixta, los tiempos indicados para cada tipo de urgencia se deben repartir entre la votación de la Comisión mixta y las votaciones posteriores en cada Cámara. De esta manera, con urgencia simple, la Comisión mixta tiene 10 días para votar el proyecto y, luego, cada Cámara tiene 10 días para despacharlo. Bajo urgencia suma, la Comisión mixta tiene cuatro días y cada Cámara tres días. Bajo urgencia inmediata, la Comisión mixta y las Cámaras tienen un día cada una para despachar el proyecto.

Si bien la Constitución estipula plazos determinados para votar los proyectos con urgencia legislativa, ésta establece que el Presidente puede introducir y retirar las urgencias a su voluntad. Esto lleva a que el principal efecto de las urgencias sea determinar el orden del día (tanto en comisiones como en sala) y enviar señales políticas de preferencias o prioridades del gobierno ${ }^{10}$, en vez de forzar la votación de proyectos de ley ${ }^{11}$. Los Reglamentos de la Cámara de Diputados y del Senado determinan que los proyectos con urgencias legislativas toman precedencia frente al resto, a menos que sean acusaciones constitucionales o proyectos relativos a la Ley de Presupuestos.

La facultad presidencial de imponer urgencias a la tramitación legislativa de proyectos de ley da un importante poder de agenda al Presidente de la República. Al contrario de lo que ocurre en Estados Unidos, donde las comisiones pueden demorar proyectos de ley como táctica legislativa para evitar que éstos sean votados en la sala, esta facultad le permite al Ejecutivo determinar la tabla de discusión de las comisiones y de la sala de ambas Cámaras, ya que los proyectos con urgencia desplazan a proyectos que no gozan de ésta en el orden del día. Esto otorga al Presidente la capacidad para determinar los ritmos de tramitación legislativa, al permitirle, por una parte, forzar la discusión de ciertos proyectos y, por otra, mantener proyectos fuera de discusión a través de la congestión de la agenda con otros proyectos de ley ${ }^{12}$.

Las urgencias legislativas también sirven como mecanismo para obtener información sobre las posturas de los parlamentarios. Al poder introducir y retirar las urgencias, se pueden testear las posturas individuales de los parlamentarios sin la necesidad de realizar votaciones adversas. Por esta razón, vemos que el Eje-

10 Entrevista con Carlos Carmona (2006).

11 La Constitución no establece lo que sucede en caso de que el proyecto de ley bajo urgencia no se vote en el tiempo indicado.

12 Según Axel Buchheister, Director del Programa Legislativo de Libertad y Desarrollo, cuando la oposición quiere ver un proyecto suyo, el Ejecutivo le pone suma urgencia o discusión inmediata a ese proyecto, de modo que el debate sea corto y no se pueda escuchar a los ciudadanos interesados: "Ese sistema se usa sistemática y constantemente como una forma de impedir el debate". 
cutivo rara vez pierde una votación. Cuando el gobierno no cuenta con los votos necesarios para aprobar un proyecto simplemente lo retira de discusión. Entre 1990 y 2006, se han rechazado sólo 18 proyectos del Ejecutivo. Esta cifra es muy pequeña si se la compara con los 79 proyectos de parlamentarios que han sido rechazados durante el mismo período.

Por último, las urgencias le otorgan una herramienta de negociación política adicional al permitirle "sacar al pizarrón" a la oposición. El Ejecutivo puede evitar pronunciarse públicamente sobre un tema que le es adverso en la opinión pública simplemente retirándolo de la agenda; por el contrario, puede exponer la postura de la oposición en temas de baja popularidad en momentos claves en la política nacional ${ }^{13}$.

Datos empíricos sobre la actividad legislativa chilena para el período 19902006 sugieren que los poderes de agenda que otorga la Constitución al Ejecutivo efectivamente le permiten controlar qué proyectos de ley se analizan durante la mayor parte del proceso legislativo. De esta manera, si bien desde 1990 el 57\% de los proyectos de ley se originó a través de mociones parlamentarias, el 68\% de estas mociones no registró avance alguno en el proceso legislativo, y sólo el 13\% de ellas se transformó en ley. En contraposición, sólo el 9\% de los proyectos de ley del Ejecutivo no registraron avance en su tramitación y el 72\% de ellos fueron promulgados como ley ${ }^{14}$.

\section{Poderes de legislación unilateral: decreto, plebiscito, y vetos}

\subsection{Facultades Para emitir DeCREtos y llamar a Plebiscito}

La facultad de gobernar por decreto es uno de los poderes más fuertes del que puede gozar un Presidente. La amplitud del poder de decreto dependerá de los requisitos para su aplicación, a saber, la existencia de requerimiento de aprobación previa del Congreso, la restricción de las materias de ley sobre las cuales se puede aplicar, el período de tiempo para aplicarlo, y la facultad del Congreso para establecer otras restricciones a su aplicación.

En el BID (2006) se argumenta que en Argentina, Brasil y Colombia el Presidente puede promulgar legislación por decreto en la mayoría de las materias, y en Ecuador y Perú en materias económicas y fiscales, respectivamente. Aunque en estos países el Congreso puede anular los decretos, esta facultad entrega un poder significativo al Presidente para influenciar la composición de la agenda legislativa. Cox y Morgenstern (2002) establecen que en Argentina, el Presidente tiene la facultad para dictar decretos sin la aprobación previa del Congreso, los cuales sólo pueden ser derogados a través de la aprobación de una ley por parte

13 Según Carlos Carmona los principales beneficios de las urgencias para el Ejecutivo son de fijar el orden del día, y de enviar señales políticas. Manifiesta, a la vez, que en la práctica la urgencia no sirve para forzar la aprobación de proyectos de ley.

14 Aninat y Londregan (2006b). 
del Congreso (la que, a su vez, puede ser vetada por el Presidente). Esto le dio un enorme poder a Menem para gobernar prescindiendo del Congreso. Morgenstern (2002) va más allá y plantea que las legislaturas de Brasil y Argentina son prácticamente impotentes respecto de los decretos del Presidente. Argumenta que el poder de decreto en estos países le da la capacidad al Presidente de gobernar con prescindencia del Congreso.

En contraste con lo anterior, en Chile la Constitución otorga facultades restrictivas de decreto al Presidente de la República. Éste puede solicitar al Congreso autorización para dictar decretos con fuerza de ley (DFL) sobre un reducido campo de materias de legislación ordinaria, por un período no superior a un año. El artículo 64 de la CPR establece explícitamente que la delegación legislativa no puede recaer sobre materias de quórum especial, entre otras restricciones. Por su parte, toda ley de autorización que apruebe el Congreso debe señalar de manera precisa las materias sobre las cuales recaerá la delegación y puede determinar a su voluntad las restricciones que estime conveniente ${ }^{15}$. Por lo tanto, si bien el Presidente chileno formalmente goza de poder de decreto, tal facultad, más que acrecentar el poder del Presidente para modificar el status quo unilateralmente, constituye una herramienta adicional de cooperación entre el Presidente y el Congreso. En Chile, el decreto se utiliza como parte de la técnica legislativa para que el Ejecutivo determine aspectos específicos de la legislación que le son propios, en razón de la mayor capacidad técnica de los ministerios con respecto a los legisladores ${ }^{16}$.

La Constitución también otorga al Presidente una facultad restringida de iniciativa para llamar a plebiscito. Esta sólo se puede utilizar para dirimir una disputa entre el Presidente y el Congreso cuando este último logra insistir exitosamente ante un veto presidencial sobre un proyecto de reforma constitucional. Si el Congreso insiste con dos tercios de sus miembros en ejercicio en el texto de reforma aprobado originalmente, el Presidente puede convocar a plebiscito para que la ciudadanía decida. Siendo ésta una situación extrema, el plebiscito nunca se ha aplicado en Chile desde la entrada en vigencia de la actual Constitución.

\subsection{Poderes De Veto}

Las Constituciones varían en cuanto a las características del poder de veto otorgado al Presidente y a las facultades del Congreso para aprobar el veto o para insistir en la promulgación de la versión del proyecto de ley sancionada por el mismo. Estas variaciones, a veces aparentemente nominales y, generalmente, ignoradas

15 En Chile el espacio Legislativo está dividido entre materias de ley y materias reglamentarias. La CPR establece explícitamente las materias de ley. En la potestad reglamentaria del Presidente de la República, éste puede dictar decretos que no son materias de ley. Además, los reglamentos del Ejecutivo deben ser aprobados por la Contraloría General de la República (organismo autónomo) antes de entrar en vigencia, y las personas tienen posibilidades de impugnar los reglamentos ante tribunales ordinarios o ante el Tribunal Constitucional.

16 Carlos Carmona (2006). 
por los estudios académicos, tienen efectos sustantivos sobre el poder real del Presidente y el balance de poder entre éste y el Congreso.

Los poderes de veto presidenciales se pueden clasificar en dos grandes categorías: los vetos absolutos (o totales) y los vetos constructivos. Los primeros permiten al Presidente rechazar el proyecto de ley aprobado por el Congreso en su totalidad, en cuyo caso se mantiene el status quo. En los segundos, el veto presidencial enmienda la ley aprobada por el Congreso pudiendo suprimir una parte del proyecto de ley o, incluso, proponer un cambio o una adición a éste. En Estados Unidos el Presidente sólo goza de veto absoluto, mientras que en América Latina los Presidentes poseen ambos poderes de veto ${ }^{17}$.

En caso del veto absoluto, el Congreso puede votar si insiste en su versión del proyecto de ley. En caso de no hacerlo, o no alcanzar el quórum necesario para ello, se da por aprobado el veto y se mantiene el status quo. En caso de insistencia, entre los países de la región existen diferentes procedimientos de acuerdo a cuál de las Cámaras del Congreso que debe decidir, y al umbral de votación necesario para insistir. En la mayoría de los países se requiere la votación concurrente de ambas Cámaras (o del Congreso unicameral), excepto en Brasil y Bolivia en que se debe realizar una votación en sesión conjunta de ambas Cámaras. Los quórum requeridos para la anulación del veto varían entre mayoría absoluta, en siete países de la región; 2/3, en 11 países; sólo en Uruguay se requiere de 3/5 de los parlamentarios ${ }^{18}$. En algunos países, esta mayoría debe ser de los miembros presentes el día de la votación y, en otros, de los miembros en ejercicio.

En el caso del veto constructivo, que puede ser supresivo o modificatorio, existen dos modelos principales de procedimiento ${ }^{19}$. En Argentina, Brasil, Uruguay y Ecuador, el Congreso puede votar su anulación pero no su aprobación. Es decir, si el Congreso no logra la mayoría necesaria para insistir en su versión del proyecto de ley vetado, se da por aprobada la versión del Ejecutivo. En el modelo de Chile, Costa Rica, México y Bolivia, sin embargo, existe un requisito de aprobación del veto constructivo por simple mayoría del Congreso, por lo que se deben realizar dos votaciones separadas para su aprobación y para su anulación. En caso de que se apruebe el veto constructivo, se promulga la versión de la ley propuesta por el Presidente; si no se logra la aprobación del veto, se mantiene el status quo; y si se logra la insistencia, se promulga la versión original aprobada por el Congreso ${ }^{20}$. Por lo tanto, bajo este modelo un Presidente sin mayoría parlamentaria no puede imponer al Congreso una versión distinta del proyecto a la aprobada por la mayoría del mismo ${ }^{21}$.

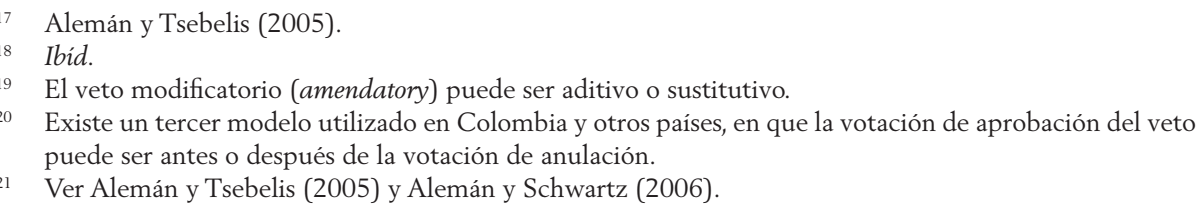


Estas diferencias de procedimiento tienen un impacto significativo en el poder presidencial para modificar el status quo unilateralmente, o bypassear al Congreso. En el caso del primer modelo, un Presidente de minoría puede imponer al Congreso su versión preferida del proyecto de ley si es que cuenta con un apoyo suficiente para evitar la insistencia parlamentaria. En el caso de Uruguay, el Presidente tiene poderes de veto aditivo, sustitutivo y supresivo, los cuales le permiten responder al Congreso con un proyecto de ley modificado a su voluntad, que este último no puede rechazar si no cuenta con el quórum necesario para la insistencia. Como consecuencia de lo anterior, el Ejecutivo puede cambiar el status quo unilateralmente mientras el Congreso no logre un voto disciplinado de tres quintos de sus miembros. De esta manera, el Presidente uruguayo puede legislar con minoría, a través del poder de veto, si cuenta con el apoyo de al menos dos quintos más uno de los votos en el Congreso. Con todo, para determinar el real poder del Presidente frente al Congreso, es preciso analizar cómo interactúan el sistema electoral y el sistema de partidos políticos con las disposiciones constitucionales. Volveremos a este punto más abajo.

En Chile, la Constitución establece que el Presidente cuenta con poderes de veto absoluto y modificatorio. Cuando el Presidente recibe un proyecto de ley aprobado por el Congreso nacional, puede vetarlo en su totalidad $u$ ofrecer un proyecto de ley modificado. La modificación puede referirse a una parte del proyecto aprobado por la Cámara (si es que es un veto supresivo) o puede tratarse de una versión modificada del mismo o, incluso, de un proyecto totalmente nuevo. En caso de un veto absoluto, el Congreso puede aceptar el veto o votar la insistencia del texto original, para lo cual requiere la votación favorable de dos tercios de los miembros presentes si se trata de legislación ordinaria y de dos tercios de los miembros en ejercicio, en caso de reforma constitucional o leyes de quórum especial.

Frente a un veto modificatorio del Presidente de la República, el Congreso debe hacer dos votaciones. En la primera, se decide la aprobación del veto por simple mayoría de los miembros presentes o por la mayoría necesaria según el quórum correspondiente a la materia de ley en cuestión ${ }^{22}$. Si no se aprueba el veto, se mantiene el status quo y, en dicho caso, el Congreso puede realizar una segunda votación para insistir en el texto original de su proyecto de ley. Para esto último, requiere cumplir con los mismos requisitos que en el caso del veto absoluto. De esta manera, en contraposición al planteamiento de Godoy (2003), el Presidente de Chile sólo puede aprobar modificaciones al status quo que cuenten con la mayoría parlamentaria requerida por la materia de ley en cuestión. Bajo este procedimiento, un Presidente sin mayoría sólo puede bloquear cambios al status quo aprobados por el Congreso, pero no legislar unilateralmente.

Por otra parte, ciertos aspectos de las facultades de veto incrementan el poder del Presidente. Debido a que en Chile los vetos presidenciales se aplican sólo a

22 Reglamento del Senado, art. 188. 
lo contenido en la observación ${ }^{23}$, si el Congreso no aprueba un veto presidencial pero tampoco logra la mayoría necesaria para la insistencia, la ley que finalmente se aprueba es la parte del proyecto sobre la cual existe acuerdo. En el extremo, tal situación puede llevar a que un Presidente de minoría fuerce la aprobación de una ley desbalanceada que sea menos preferida que el status quo para la mayoría del Congreso. Sin embargo, el Reglamento de la Cámara de Diputados establece explícitamente que si una disposición principal del proyecto es vetada, las disposiciones accesorias a éste quedan también sin efecto ${ }^{24}$.

Bajo un sistema de veto en que se requiere de una mayoría parlamentaria (simple o calificada) para la aprobación de vetos modificatorios, el sistema electoral y el sistema de partidos juegan un rol particularmente relevante. El sistema electoral binominal que induce a la formación de dos coaliciones parlamentarias estables y disciplinadas, otorga al Ejecutivo un contingente parlamentario que oscila entre el $40 \%$ y $60 \%$ de los miembros de ambas cámaras. En este contexto, si el Presidente es de minoría, no puede introducir vetos constructivos. Pero, si es de mayoría, sólo puede aprobar vetos constructivos en materias de ley ordinarias o de quórum calificado (mayoría absoluta de los miembros en ejercicio), pero no en las materias de ley más trascendentes que requieren de 4/7, 3/5, o 2/3 de los parlamentarios en ejercicio.

De esta manera, el Presidente de Chile cuenta con poderes de veto significativos pero más débiles que muchos de los otros Presidentes de la región. $\mathrm{Si}$, como medida del poder del Presidente, sólo se considera a los quórum requeridos para la insistencia parlamentaria de la versión del proyecto de ley aprobada por el Congreso y vetado por el Presidente -como lo hace el Compendio Estadístico de PNUD ${ }^{25}$-, no es posible apreciar el real balance de poder entre el Presidente y el Congreso. Este estudio considera al Presidente chileno con un poder de veto mayor que el que posee su símil uruguayo, porque el quórum requerido para la insistencia en dicho país es de $3 / 5$ comparado con los $2 / 3$ que se requieren en Chile. Sin embargo, como se ha argumentado, en Uruguay el Presidente puede imponer un veto constructivo modificatorio, aun contra la voluntad de la mayoría de los parlamentarios. En Chile, en cambio, eso no es posible.

\section{INFLUENCIA DE LA LEGISLATURA}

La influencia de la legislatura sobre el proceso legislativo y el balance de poder entre el Presidente y el Congreso, depende de las facultades constitucionales del Congreso; de su estructura, procedimientos y capacidad técnica; y del balance de fuerzas partidistas de los parlamentarios ${ }^{26}$.

23 El Presidente puede determinar qué se incluye bajo una observación: puede ser una parte de un inciso, un inciso, un artículo o varios artículos.

24 Reglamento de la Cámara de Diputados, art. 172.

25 Ver PNUD (2005).

26 BID (2006), Morgenstern y Naciff (2002) y Saiegh (2005). 
En Chile, en un contexto de dominio del Presidente de la República sobre la agenda legislativa, el Congreso nacional tiene diversas atribuciones y características que le permiten jugar un rol clave en el proceso legislativo. El sistema Legislativo basado en un Congreso bicameral con dos o tres trámites legislativos, con uso extensivo de comisiones, con quórum especiales para las materias de ley de mayor trascendencia, junto a la existencia de un Tribunal Constitucional independiente, refuerzan el poder del Congreso.

\section{Estructura del Congreso, quórum, y proceso de enmienda}

\subsection{ESTRUCTURA DE LA LEGISLATURA Y RELEVANCIA DE LOS QUÓRUM ESPECIALES}

En el proceso legislativo chileno, los proyectos de ley deben ser aprobados por ambas Cámaras del Congreso, en lo que se denomina trámites constitucionales. Los proyectos de ley se pueden introducir tanto por el Senado como por la Cámara de Diputados, y ellos deben ser aprobados en forma idéntica por la Cámara de origen y por la Cámara revisora. Si ello no es así, existe un tercer trámite constitucional en que la Cámara de origen vota bajo close rule el proyecto aprobado por la otra Cámara. Si no hay acuerdo, se forma una Comisión mixta con miembros de ambas Cámaras para resolver las diferencias. En caso de acuerdo, ambas Cámaras deben ratificarlo.

En cada Cámara los proyectos tienen dos lecturas, llamadas trámites legislativos, en que se discuten y votan los proyectos en general y, luego, en particular. En cada trámite los proyectos de ley se envían a una o más comisiones para que los analicen y, a través de un informe, propongan un texto para ser considerado por la sala de la Cámara. Si un proyecto de ley implica gastos para el Estado, la Comisión de Hacienda debe conocerlo necesariamente además de la comisión temática encargada de la materia de ley en cuestión. La regla general de votación es close rule para la votación en general y open rule para la votación en particular. En el primer caso, la sala vota el proyecto como un todo para decidir sobre la idea de legislar, mientras que en la votación en particular la sala vota artículo por artículo el texto propuesto por la comisión. Sólo por unanimidad de los jefes de bancada se puede acordar la votación en sala de un proyecto de ley bajo close rule.

Una variable clave en el poder del Congreso frente al Presidente de la República es la existencia de quórum especiales para la aprobación de materias de ley de mayor trascendencia, de leyes interpretativas de la Constitución y para las reformas constitucionales ${ }^{27}$. La Constitución contempla cuatro quórum diferentes para la aprobación de proyectos de ley. La legislación ordinaria necesita de mayoría simple de los parlamentarios presentes; las leyes de quórum calificado requieren de la mayoría de los parlamentarios en ejercicio; las leyes orgánicas constitucionales requieren de las cuatro séptimas partes de los parlamentarios en ejercicio para su aprobación; y las leyes interpretativas de la constitución deben

\footnotetext{
27 Aninat (2006).
} 
ser aprobadas por tres quintos de los parlamentarios en ejercicio ${ }^{28}$. Los proyectos de reforma constitucional requieren de la aprobación de tres quintas partes, o de los dos tercios de los parlamentarios en ejercicio, dependiendo del capítulo de la Constitución en cuestión ${ }^{29}$.

Los procedimientos de modificación y aprobación de proyectos de ley vigentes en Chile realzan la importancia de los quórum especiales. Las votaciones de materias de quórum especial deben realizarse artículo por artículo, y la clasificación del quórum de aprobación está determinada por la materia de ley del artículo en cuestión, por lo que un proyecto de ley puede requerir de diferentes quórum para aprobar sus distintos artículos. Por otra parte, en la discusión en particular, tanto los artículos que no reciben indicaciones como las indicaciones que son ratificadas por la unanimidad de la comisión, se dan por aprobados en la sala. Sin embargo, los artículos sujetos a quórum especial deben votarse individualmente en la comisión y en la sala. De esta manera la existencia de quórum especiales exige al gobierno alcanzar acuerdos más amplios para la aprobación de proyectos de ley en materias afectas a dichos quórum. Al empoderar al Congreso frente al Ejecutivo, esta circunstancia constituye una instancia adicional de cooperación entre ambos poderes del Estado.

\subsection{EFECTOS DEL PROCESO DE ENMIENDA SOBRE LOS RESULTADOS DEL PROCESO LEGISLATIVO}

La estructura y las reglas que determinan el proceso de introducción y aprobación de indicaciones a los proyectos de ley en tramitación legislativa tienen efectos sustantivos sobre el contenido de las leyes aprobadas y sobre el balance de poder entre el Ejecutivo y el Congreso. Estos procedimientos determinan los períodos en los cuales se pueden introducir indicaciones; el poder de las comisiones frente al resto de los parlamentarios; el poder del Ejecutivo frente a las comisiones y parlamentarios; y los contenidos de las indicaciones a través de las estrategias legislativas de cada uno de estos actores, impactando en último término en el contenido mismo de las indicaciones y del texto de los proyectos de ley finalmente aprobados.

Los principales factores que inciden en este proceso son: la existencia de restricciones de tiempo para la introducción de indicaciones, de requisitos especiales para la introducción de indicaciones en sala, la posibilidad de respuesta de las comisiones a dichas indicaciones, el tipo de "agenda de votación" utilizada, y el orden de votación de las indicaciones. La existencia de períodos de introducción de indicaciones y de restricciones a la introducción de las mismas refuerza el poder de los miembros de comisiones y a los líderes de los partidos de la mayoría, al darles tiempo para responder a dichas indicaciones. En países como Argentina o México no existen tales restricciones. En Chile, en cambio, los parlamentarios sólo pueden presentar indicaciones a los proyectos de ley 
durante su discusión en particular en la comisión. En sala no se pueden introducir indicaciones nuevas. Sólo pueden re-introducir indicaciones, que hayan sido previamente rechazadas en comisión, el Presidente de la República, dos jefes de comité (bancada) en la Cámara de Diputados, o parlamentarios que cuenten con la firma de diez senadores en la Cámara alta ${ }^{30}$.

En los países en que se permite la introducción de indicaciones en sala, a veces se permite que la comisión que informó el proyecto responda con una contrapropuesta a dicha indicación ${ }^{31}$. Esta es una facultad relevante ya que otorga el beneficio de última movida, el cual implica un mayor poder de negociación para la comisión en las etapas previas de introducción de indicaciones. En Chile, dado que no se permite la introducción de indicaciones nuevas en sala, las comisiones tampoco pueden introducir contrapropuestas en dicha instancia ${ }^{32}$.

El método de votación empleado para aprobar indicaciones y el orden en que éstas se voten produce efectos directos en las estrategias legislativas de los distintos actores involucrados en el proceso, y puede afectar el contenido de los proyectos de ley. Los dos principales métodos de votación que existen son el utilizado en Inglaterra y sus ex colonias, llamado amendment, y el utilizado en Europa continental y en Latinoamérica, llamado de eliminación secuencial ${ }^{33}$. En el primer caso, las indicaciones alternativas que recaen sobre un mismo artículo se votan en pares unas contra otras y, al final, se vota el texto aprobado contra el status quo. En el sistema utilizado en Latinoamérica las indicaciones se votan secuencialmente contra el status quo en un orden predeterminado, y cuando una indicación es aprobada, ella se convierte en el resultado final en esa materia y las demás se consideran rechazadas. Bajo este método, el orden de votación es muy importante, ya que mientras antes se vote una indicación mayor será la posibilidad de que sea aprobada. Si una indicación tiene mayoría absoluta de las preferencias de la sala o es un ganador de Condorcet, dicha indicación será elegida bajo cualquier método y en cualquier orden de votación. Sin embargo, si existe intransitividad colectiva, tanto el procedimiento como el orden de votación son determinantes para la decisión que adopte la sala.

En Chile, el orden de votación de indicaciones mutuamente excluyentes lo determina el Presidente de la Cámara en cuestión ${ }^{34}$. No existen disposiciones legales al respecto, pero la norma informal contempla que se votan primero las indicaciones del Ejecutivo, luego las de los parlamentarios y, al final, el texto propuesto por la comisión. Bajo el sistema de votación de eliminación secuencial,

30 Reglamento de la Cámara de Diputados, art.131.

31 Alemán (2003).

32 Sólo la Comisión de Hacienda puede introducir indicaciones nuevas en sala. Esta comisión conoce los proyectos de ley correspondientes a otras comisiones cuando los proyectos tienen implicancias financieras para el Estado.

33 Alemán (2003).

34 Reglamento de la Cámara de Diputados, art.149. 
este orden refuerza el poder presidencial ya que las probabilidades de adopción de una indicación aumentan mientras antes se la vote ${ }^{35}$.

El orden de votación también produce un efecto sustantivo en las indicaciones que se presentan. Si un actor sabe que su indicación será votada al final, ésta debe contar con mayoría absoluta o al menos ser un ganador de Condorcet; en cambio, si la indicación es votada primero ésta sólo debe ser preferible a la alternativa con la cual será comparada. En agendas de votación de eliminación secuencial un actor racional sabe que la alternativa real a la indicación que se está votando no es el status quo, sino alguna de las alternativas siguientes que vencerá al status quo; por lo tanto, es como si se votara contra ella. Así, dado que en Chile sólo se requiere de un mínimo de apoyo parlamentario o de la voluntad del Ejecutivo para reintroducir en la sala una indicación rechazada en comisión, la propuesta de la comisión debe ser una ganadora de Condorcet para que sea seleccionada ${ }^{36}$. Si no, una de las indicaciones que se vote antes de ella será seleccionada, y la indicación de la comisión no se votará. Esto modifica sustantivamente el tipo de indicaciones que se presentan y aprueban en comisión.

En suma, las reglas y procedimientos del proceso de enmienda en su conjunto fortalecen a las comisiones en relación al resto de los legisladores, y refuerzan el poder constitucional del Presidente en relación al Congreso. El poder para reintroducir en sala indicaciones rechazadas en comisión, y para que éstas sean votadas primero y bajo los tiempos determinados por el propio Ejecutivo a través de urgencias legislativas, aumenta considerablemente la probabilidad de que la sala adopte la versión preferida por el Presidente por sobre el texto propuesto por la comisión.

\section{Determinantes de la capacidad legislativa del Congreso, $y$ sus efectos}

La capacidad legislativa del Congreso depende principalmente de la carrera legislativa de los parlamentarios (tasa de reelección) y de la eficiencia del sistema de comisiones. El BID (2006) establece que las reglas electorales y el grado de centralización en los procesos de nominación de candidatos pueden afectar las ambiciones e incentivos profesionales de los legisladores, así como su experiencia. La baja tasa de reelección en Latinoamérica lleva a los legisladores a buscar una carrera fuera de las legislaturas, teniendo como resultado que los objetivos profesionales de los legisladores estén enfocados a satisfacer más a los líderes de los partidos que a los intereses y demandas de los ciudadanos.

Para los estándares latinoamericanos, el Congreso chileno tiene alta capacidad legislativa ${ }^{37}$. A diferencia de países como México en que los legisladores tienen que dejar el Congreso después de un período, o Argentina en que el futuro de un

\footnotetext{
Farquharson (1969) lo demostró para el caso general de votaciones en eliminación secuencial.

Ver Alemán (2003).

Aninat et al. (2007).
} 
legislador está en manos de los jefes de partidos a nivel provincial, en Chile los legisladores hacen carrera en el Congreso. El sistema electoral vigente y la ausencia de límites a la reelección han llevado a que los parlamentarios chilenos tengan la más alta tasa de reelección de la región (60\% en la Cámara de Diputados). Lo anterior conduce a que los parlamentarios dominen los intrincados procedimientos legislativos, permitiéndoles un mayor poder de negociación con el Ejecutivo y una mayor capacidad de aprobar legislación de mejor calidad.

Otro aspecto fundamental en la capacidad legislativa de los Congresos radica en las características del sistema de comisiones. Es en esta instancia donde los ciudadanos afectados por la legislación pueden representar sus intereses y puntos de vista, y también donde el Ejecutivo presenta la evidencia y argumentación para respaldar sus proyectos de ley. Por lo tanto, es aquí donde se producen las discusiones técnicas y donde se introducen la gran mayoría de las indicaciones tanto del Ejecutivo como de los parlamentarios. La capacidad legislativa del sistema de comisiones está determinada por el número de comisiones por legislador; el grado de correspondencia entre las jurisdicciones de las comisiones y los ministerios del Estado (esto permite un mayor grado de coordinación y, por ende de eficiencia, en el trabajo entre la legislatura y el Ejecutivo); y las reglas que determinan cómo se eligen los miembros y los Presidentes de las comisiones, así como la frecuencia en la rotación de sus miembros (mientras mayor sea dicha frecuencia, menor será el nivel de especialización de sus miembros) ${ }^{38}$.

La Constitución chilena sólo establece la existencia de las Comisiones de Hacienda en cada Cámara ${ }^{39}$. Sin embargo, los reglamentos de las Cámaras establecen la existencia de diez y nueve comisiones permanentes, y permiten el establecimiento de comisiones especiales. Las comisiones en la Cámara de Diputados están compuestas por trece miembros y en la Cámara alta por cinco senadores. El promedio de comisiones por legislador en Chile es dos, lo que permite que éstos puedan especializarse en las materias que les corresponden. Del total de ministerios del Estado, sólo dos (Secretaría General de Gobierno y Secretaría General de la Presidencia) no tienen una comisión específica como contraparte en la legislatura, por lo que el grado de coordinación entre el Ejecutivo y las comisiones es alto para los estándares latinoamericanos. Los miembros de las comisiones son elegidos por la Cámara a propuesta de la Mesa Directiva y el Reglamento de la Cámara establece que la composición partidista de las comisiones debe ser proporcional a la representación que tiene cada partido dentro de la Cámara. En la práctica, sin embargo, los partidos intercambian puestos dentro de las comisiones que consideran estratégicas. Los Presidentes

38 Saiegh (2005) y BID (2006).

39 Esta comisión está encargada de informar los proyectos en lo relativo a su incidencia en materia presupuestaria o financiera del Estado, de sus organismos o empresas. La comisión debe indicar en sus informes la fuente de los recursos efectivos con que se propone atender el gasto que signifique el proyecto y la incidencia de sus normas en la economía del país (art. 17 ley orgánica constitucional del Congreso). 
de las comisiones (que tienen atribuciones para declarar la admisibilidad de las indicaciones) son elegidos por la mayoría de los miembros de las mismas.

\section{EFECTOS DEL SISTEMA DE PARTIDOS EN EL PROCESO LEGISLATIVO}

\section{Balance de fuerzas partidarias en el Congreso y el contingente partidario del Presidente}

Por último, el contingente legislativo con que cuenta el Presidente de la República en el Congreso, así como la unidad y disciplina que logre la oposición son fundamentales para determinar el balance de poderes entre el Congreso y el Presidente de la República. Los poderes partidarios no sólo afectan la capacidad del Ejecutivo para formar mayorías, sino también afectan la incidencia de los quórum especiales para ciertas materias de ley, la efectividad de las facultades de veto del Presidente, y las posibilidades de obtener la delegación legislativa por parte del Congreso para dictar decretos con fuerza de ley.

El sistema binominal vigente en Chile organiza a los partidos con representación parlamentaria en dos grandes coaliciones estables y disciplinadas. El sistema electoral es proporcional, con dos escaños por distrito; utiliza listas abiertas y la cifra repartidora D'Hont para la asignación de escaños; permite coaliciones electorales vinculantes a nivel nacional; y cada lista puede presentar dos candidatos como máximo en cada distrito. Esto lleva a que a nivel distrital las dos primeras mayorías obtienen un escaño cada una, a menos que la primera doble la cantidad de votos obtenida por la segunda. La consecuencia de esto es que si una lista obtiene el 33\% de los sufragios, ella asegura el 50\% de los escaños en ese distrito.

El conjunto de disposiciones del sistema electoral incentiva a los partidos a formar coaliciones nacionales que se organizan de manera a intentar doblar a (y evitar ser doblados por) la otra lista más votada en la cantidad de votos obtenidos por distrito. Desde 1990, en el 85\% de los distritos de la Cámara de Diputados y el $95 \%$ de las circunscripciones senatoriales, cada coalición ha obtenido el $50 \%$ de los escaños, llevando a que los bloques políticos hayan logrado una representación que fluctúa entre el 40\% y el 60\% de los escaños de cada Cámara del Congreso. El elevado costo que potencialmente significaría para un partido abandonar una coalición unilateralmente (ya que si el partido no logra el umbral del 33\% de los sufragios no obtiene representación parlamentaria alguna), induce un comportamiento legislativo cohesionado y disciplinado por parte de los legisladores de cada coalición. La restricción del número de candidatos a presentar por lista en cada distrito implica que las coaliciones compuestas por más de dos partidos deben negociar los cupos en la lista de cada distrito para las elecciones legislativas. Estas constantes negociaciones intra-coalición refuerzan la disciplina de sus miembros ${ }^{40}$.

40 Ver Carey (2002), el alto nivel de disciplina en la votación de las coaliciones hace que la unidad de análisis más relevante en el Congreso chileno sea la coalición más que el partido. 
Esta estructura del sistema de partidos es fundamental en el equilibrio de poderes entre el Presidente y el Congreso en Chile. Bajo este sistema, el Presidente cuenta siempre con un respaldo cercano al 50\% de los parlamentarios en ejercicio y debe enfrentar una oposición cohesionada y estable. En caso de ser un Presidente de mayoría, éste sólo debe ordenar los votos de su coalición para conseguir la aprobación de legislación sobre materias de ley de quórum simple y calificado, obtener la aprobación de vetos constructivos y la autorización para dictar decretos en dichas materias de ley. Sin embargo, para lograr la aprobación sobre materias de ley de quórum más elevado o de vetos constructivos, así como la autorización para dictar decretos sobre esas materias, necesariamente debe obtener el concurso de la oposición. En este contexto, si bien el Presidente chileno tiene importantes poderes de agenda, el Congreso es un actor fundamental del proceso legislativo ${ }^{41}$.

\section{Conclusión}

Los estudios sobre legislaturas latinoamericanas han tendido a sobreestimar los poderes legislativos del Presidente chileno. Este último tiene amplios poderes de agenda pero no cuenta con facultades para gobernar con prescindencia del Congreso, como ocurre en otros países de la región. El Presidente de Chile goza del control de la agenda legislativa a través de amplias facultades en la aplicación de urgencias, y de su iniciativa exclusiva sobre diversas materias de ley (que incluyen control sobre el presupuesto y la mayor parte de la política económica). Sin embargo, el Ejecutivo chileno goza de un poder de plebiscito extremadamente restringido, y los poderes de veto y de decreto figuran entre los más débiles de la región.

Por otra parte, una variable fundamental en la determinación del rol que juega el Congreso en Chile, son los quórum especiales que obligan al Presidente a negociar permanentemente tanto con los parlamentarios de oposición como con sus adherentes. El poder del Congreso se ve reforzado por el extenso y complejo proceso de enmienda, el cual otorga numerosas instancias a los legisladores para demorar la tramitación de los proyectos de ley, debido a la alta capacidad legislativa del Congreso y a la independencia de los parlamentarios respecto del Presidente de la República.

El sistema electoral vigente en Chile y la ausencia de límites a la reelección, junto a otros incentivos del sistema de partidos, producen las más altas tasas de reelección parlamentaria en Latinoamérica, lo que unido a un sistema de comisiones eficiente ha permitido el desarrollo de carreras parlamentarias prolongadas con especialización por materias de ley. Asimismo, el sistema electoral binominal vigente en Chile

${ }^{41}$ El bajo grado de competencia y las altas barreras a la entrada a nuevas fuerzas políticas que produce el sistema binominal afectan al sistema político negativamente. El análisis de éstos y otros problemas de este sistema electoral van más allá del alcance de este artículo. 
está intrínsecamente relacionado con el balance de poderes entre el Ejecutivo y el Legislativo. El sistema binominal induce a la formación de un sistema de partidos que otorga al Presidente un contingente Legislativo disciplinado que oscila entre el 40 y el 60\% de ambas Cámaras del Congreso. Pero a la vez, crea una oposición ordenada y con un contingente Legislativo superior a los quórum requeridos para las reformas legislativas de importancia. De esta manera, el Presidente de la República sólo puede avanzar su agenda legislativa en materias de legislación ordinaria si cuenta con mayoría en ambas Cámaras del Congreso. En cambio, para materias de quórum calificado las cuales, como hemos visto, en Chile son muy amplias necesita el concurso de la oposición ${ }^{42}$. En un sistema de interacción repetida entre los actores políticos, el Ejecutivo debe negociar de manera permanente con la oposición, incluso cuando no necesita de sus votos, para lograr la concurrencia de ésta en la aprobación de la agenda legislativa del Presidente en materias sujetas a quórum especial.

El caso chileno, hasta ahora considerado paradójico por su alto grado de cooperación política en presencia de un presidencialismo fuerte, ilustra la necesidad de avanzar hacia un marco de análisis que permita comprender mejor el rol de las legislaturas en América Latina y el poder de éstas en relación al Ejecutivo. En este trabajo se intenta contribuir a esclarecer las variables institucionales que favorecen el desarrollo de un proceso legislativo cooperativo y las que, por el contrario, pueden inducir a desestabilizar el sistema político.

\section{Bibiliografía}

Alemán, Eduardo. 2003. "Legislative Rules and the Amending Process: Theory and Evidence from Argentina, Chile, and Mexico". Artículo presentado en The American Political Science Association Annual Meeting, Agosto 28-31, Filadelfia, Pennsylvania.

Alemán, Eduardo. Enero 2006. "Policy Gatekeepers in Latin American Legislatures". Latin American Politics and Society 48 (3): 125-155.

Alemán, Eduardo; Schwarts, Thomas. 2006. "Presidential Vetoes in Latin American Constitutions". Journal of Theoretical Politics 18 (1): 98-120.

Alemán, Eduardo; Tsebelis, George. Abril 2005. "Presidential Conditional Agenda Setting in Latin America". World Politics 57 (3): 396-420.

Aninat, Cristóbal. 2006. "E1 Proceso Legislativo Chileno: Análisis del Proyecto de Ley de Rentas Vitalicias 1994-2004”. Documento de Trabajo Departamento de Estudios de Superintendencia de Administradoras de Fondos de Pensiones de Chile.

Aninat, Cristóbal; Londregan, John. 2005. "Urgencies, Gate Keeping and Agenda Control in the Chilean Congress". Working Paper No. 11, Center on Institutions and Governance, University of California at Berkeley, Berkeley, California.

Aninat, Cristóbal; Londregan, John. 2006. "Measuring Spatial Aspects of Legislative Delay". Working Paper No. 26, Center on Institutions and Governance, University of California at Berkeley, Berkeley, California.

42 Aquí no se hace un juicio sobre los méritos o defectos del sistema electoral binominal, sino que se establece explícitamente que el sistema electoral es parte constitutiva del diseño institucional que rige el proceso legislativo en Chile. 
Aninat, Cristóbal; Londregan, John. 2006b. Base de datos del proceso Legislativo chileno, legislaturas 1990-1994, 1994-1998, 1998-2002 y 2002-2006.

Aninat, Cristóbal; Londregan, John; Navia, Patricio; Vial, Joaquín. 2007. "Political Institutions, Policymaking Processes and Policy Outcomes in Chile". Por publicarse en E. Stein y M. Tommasi (eds.), Political Institutions, Policymaking Processes, and Economic Policy in Latin America. Stanford University Press.

BID (Banco Inter-Americano del Desarrollo). 2006. "The Politics of Policies. Economic and Social Progress in Latin America 2006 Report". Washington D.C.: BID.

Buchineister, Axel. 2006. Director del Programa Legislativo de Libertad y Desarrollo. Entrevista con Cristóbal Aninat y Valeria Palanza. Santiago, Chile.

Carey, John. 2002. "Parties, Coalitions, and the Chilean Congress in the 1990s". En Scott Morgenstern y Benito Nacif (eds.), Legislative Politics in Latin America. Cambridge: Cambridge University Press, 222-253.

Carey, John; Baldez, Liza. 1999. "Presidential Agenda Control and Spending Policy: Lessons from General Pinochet's Constitution”. American Journal of Political Science 43: 29-55.

Carmona, Carlos. 2003. Director División jurídica legislativa de la Secretaría General de la Presidencia de Chile desde 1990-presente. Entrevista con Cristóbal Aninat, John Londregan y Joaquín Vial. Santiago, Chile.

Carmona, Carlos. 2006. Director División jurídica legislativa de la Secretaría General de la Presidencia de Chile desde 1990-presente. Entrevista con Cristóbal Aninat y Valeria Palanza. Santiago, Chile.

Constitución Política de la República de Chile. 2006. Versión actualizada.

Cox, Gary; Morgenstern, Scott. 2002. "Epilogue: Latin America's Reactive Assemblies and Proactive Presidents". En Scott Morgenstern y Benito Nacif (eds.), Legislative Politics in Latin America. Cambridge: Cambridge University Press, 446-492.

Farquharson, Robin. 1969. "Theory of Voting”. New Heaven: Yale University Press.

Godoy, Oscar. 2003. "parlamento, Presidencialismo y Democracia Protegida". Revista Ciencia Política 23 (2): 7-42.

Huneeus, Carlos; Berrios, Fabiola. "El Congreso en el Presidencialismo. El Caso de Chile". Santiago de Chile: CERC. Disponible en: http://www.cerc.cl/Publicaciones/El\%2 0Congreso\%20Nacional\%20chileno.pdf

Llanos, Mariana; Nolte, Detlef. 2006. "Los Congresos en América Latina: Legislaturas Reactivas, Potencialmente Activas". Documento de trabajo, German Institute for Global and Area Studies. Hamburgo.

Londregan, John. 2000. Legislative Institutions and Ideology in Chile's Democratic Transition. New York: Cambridge University Press.

Mainwaring, Scott; Shugart, Matthew. 1997. Presidentialism and Democracy in Latin America. New York: Cambridge University Press.

Morgenstern Scott. 2002. "Explaining Legislative Politics in Latin America". En Scott Morgenstern y Benito Nacif (eds.), Legislative Politics in Latin America. Cambridge: Cambridge University Press, 13-445.

Morgenstern, Scott; Naciff, Benito. 2002. Legislative Politics in Latin America. Cambridge: Cambridge University Press.

Nolte, Detlef. 2003. "El Congreso Chileno y su Aporte a la Consolidación de la Democracia". Revista de Ciencia Política, 23 (2): 43-67.

PNUD. 2005. "La democracia en América Latina. Compendio estadístico".

Reglamento de la Cámara de Diputados. Congreso Nacional de Chile.

Reglamento del Senado. Congreso Nacional de Chile.

Saiegh, Sebastián. 2005. "The Role of Legislatures in the Policymaking Process". Artículo preparado para el Banco Inter-Americano del Desarrollo. "Workshop on State Reform, 
Public Policies, and Policymaking Processes", 28 de febrero-2 de marzo, Washington, DC.

Shugart, Matthew; Carey, John. 1992. Presidents and Assemblies: Constitutional Design and Electoral Dynamics. New York: Cambridge University Press.

Siavelis, Peter. 2002. The President and Congress in Postauthoritarian Chile. Institutional Constraints to Democratic Consolidation. Pennsylvania: University Park.

Siavelis, Peter. 2002. "Exaggerated Presidentialism and Moderate Presidents: ExecutiveLegislative Relations in Chile". En Scott Morgenstern y Benito Nacif (eds.), Legislative Politics in Latin America. Cambridge: Cambridge University Press, 79-111.

Spiller, Pablo; Stein, Ernesto; Tommasi, Mariano. 2003. "Political Institutions, Policy making Processes, and Policy Outcomes: An Intertemporal Transactions Network" Design Paper $\mathrm{N}^{\circ} 1$ for the Research Network Project "Political Institutions, Policymaking Processes and Policy Outcomes". Banco Inter-Americano del Desarrollo, Washington DC. 